\title{
Lupus Mastitis in a Young Female Mimicking a Breast Carcinoma; a Rare Entity Through a Case Report and Review of the Literature
}

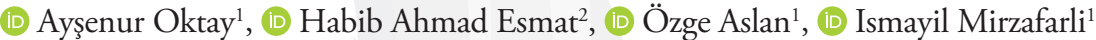 \\ ${ }^{1}$ Department of Radiology, Ege University Hospital, Faculty of Medicine, İzmir, Turkey \\ ${ }^{2}$ Department of Radiology, Kabul University of Medical Sciences, Kabul, Afghanistan
}

\begin{abstract}
Lupus mastitis (LM) is a rare presentation of lupus panniculitis (LP) that usually affects women of childbearing age and may mimic malignancy. The condition is recurrent and progresses along with the underlying disease. Breast pathology that may be associated with LM includes fat necrosis, calcification, fibrosis, scarring, and breast atrophy. Therefore, LM should be considered in the differential diagnosis of a suspicious breast mass on mammography or ultrasound, particularly if the patient has a background of systemic lupus erythematosus (SLE) or discoid lupus erythematosus (DLE). Traumatic procedures such as surgery or biopsy may worsen the condition and it is advisable to avoid biopsy if the diagnosis can be established through accurate patient history, with identification of typical clinical and radiological features. Thus, awareness of the radiologic and clinical features of LM is essential to avoid unnecessary interventional procedures that carry the potential for disease exacerbation. The authors present here the imaging findings of LM in a 37-year old female with SLE, which presented as bilateral palpable breast lumps.
\end{abstract}

Keywords: Lupus mastitis, panniculitis, lupus erythematosus, breast calcification

Cite this article as: Oktay A, Esmat HA, Aslan Ö, Mirzafarli I. Lupus Mastitis in a Young Female Mimicking a Breast Carcinoma; a Rare Entity Through a Case Report and Review of the Literature. Eur J Breast Health 2022; 18(1): 13-15

\section{Key Points}

- Lupus mastitis is a rare presentation of lupus panniculitis that usually affects women of childbearing age.

- Lupus mastitis should be considered in the differential diagnosis of a suspicious breast mass on mammography or ultrasound, particularly if the patient has a background of systemic lupus erythematosus.

- Accurate patient history and knowledge of the typical imaging appearance on ultrasound and mammogram can help the diagnosis.

- It is advisable to avoid biopsy and surgical intervention if the diagnosis of lupus mastitis can be established with the clinical and radiological features.

\section{Introduction}

Lupus mastitis (LM) is a rare manifestation of lupus panniculitis (LP), an unusual clinicopathologic variant of systemic lupus erythematosus (SLE) or discoid lupus erythematosus (DLE) that is characterized by an inflammatory process involving subcutaneous fat (1). LM is the term for breast involvement in LP and may often mimic malignancy. The condition is recurrent and progresses along with the underlying disease. Breast pathology that may be associated with LM includes fat necrosis, calcification, fibrosis, scarring, and breast atrophy (2). LM should be considered in the differential diagnosis of a suspicious breast mass on mammography or ultrasound, particularly if the patient has a background of SLE/DLE (3). Diffuse, bilateral calcifications on mammography, mainly related to calcified foci of degenerated or necrotic fat tissue, and sonographic findings of recurrent breast lumps support the diagnosis of LM. In addition, evidence of necrosis in adipose tissue and peri-glandular or perivascular lymphocyte infiltrations on the histological examination, contribute to substantiating the diagnosis (4). Herein, the authors present a rare case of lupus mastitis in a 37-year old female with a known history of SLE, which presented as bilateral palpable breast lumps and diffuse calcification on the mammogram.

\section{Case Presentation}

A 37-year-old female presented with palpable nodular masses in her breasts. She had no personal or family history of breast complaints. However, her mother had a diagnosis of familial Mediterranean fever and two cousins were diagnosed with SLE. Around four years earlier the 
index case had joint pain, facial redness, hair loss, and swelling of the eyelids, hands, and feet that had been diagnosed as having a probable connective tissue disease. On physical examination, malar rash, hyperemic lesions around the mouth, and mild eyelid and pretibial edema were noted. There were some local areas of hair loss that also involved the eyebrows. Other findings were unremarkable.

Laboratory results included: hemoglobin $(\mathrm{Hb}): 10.9 \mathrm{~g} / \mathrm{dL}$ [normal range (NR): 12-16]; white blood cells (WBC): 2,690/mm3 (NR: 4,500-11,000) with 53.5\% neutrophils; platelets $127,000 / \mathrm{mm} 3$; urea $17 \mathrm{mg} / \mathrm{dL}$; creatinine $0.58 \mathrm{mg} / \mathrm{dL}$; fasting blood glucose $91 \mathrm{mg} / \mathrm{dL}$; hemoglobin A1c (HbA1c) 5.6\%; and 24-hour urine protein $1.36 \mathrm{gr} /$ day. The anti-ribosomal and anti-nucleosome antibodies were positive but other lupus antibodies were negative. Her renal biopsy revealed type-5 lupus nephritis.

On sonographic examination, axillary lymphadenopathy and illdefined isoechoic masses, with acoustic shadows related to coarse dystrophic calcifications in the breast parenchyma, were observed. These findings were compatible with fat necrosis (Figures 1 and 2). On the mammogram, diffuse calcifications starting under the skin and scattered bilaterally in the whole of the breast parenchyma were observed. These diffuse calcifications had a coarse and curvilinear shape, consistent with the fat necrosis (Figure 3).

The patient was diagnosed with SLE, complicated by lupus nephropathy and LM. She was treated with steroids and antimalarial drugs. She was also advised to undergo ophthalmic examination. Follow-up was arranged as regular outpatient visits.

\section{Discussion and Conclusion}

Kaposi first proposed the term "lupus panniculitis" in 1883. It affects both sexes, but $90 \%$ of cases occur in women of childbearing age (2). LM is a rare, benign inflammation of the deep subcutaneous adipose tissues of the breast, seen in around $2 \%-3 \%$ of SLE patients and is rarely the initial presentation of $\operatorname{SLE}(5,6)$. LM is part of the presentation of lupus panniculitis but is termed "lupus mastitis" when the breast glands are involved. To date, only 27 cases have been reported. Kinonen et al. (6) reviewed 22 cases and six additional cases have since been reported.

The precise pathophysiology of LP/LM remains unclear, though the predominant theory suggests an autoimmune-related etiology, in keeping with the known mechanisms in SLE and DLE. Supportive evidence for this theory includes the identification of immune complexes, both at the basement membrane of the dermal-epidermal junction and in blood vessels in areas of panniculitis. In addition, there is often a marked improvement of symptoms with immunosuppressive therapy (1).

The manifestations of LM include masses in the breast, axillary lymphadenopathy, fat necrosis, fibrosis, and calcifications (7). Our patient had these typical findings.

LM may mimic breast malignancy (2). Although rare, LM should be considered in the differential diagnosis of a suspicious breast mass on mammography or ultrasound, particularly if the patient has a background of SLE/DLE (3). Common mammographic findings include ill-defined, dense breast tissue with or without associated microcalcifications. Alternatively, there may be coarse, or curvilinear calcifications in the breast tissue, suggesting fat necrosis. Ultrasound may show a similarly ill-defined, isoechoic, or hyperechoic mass.

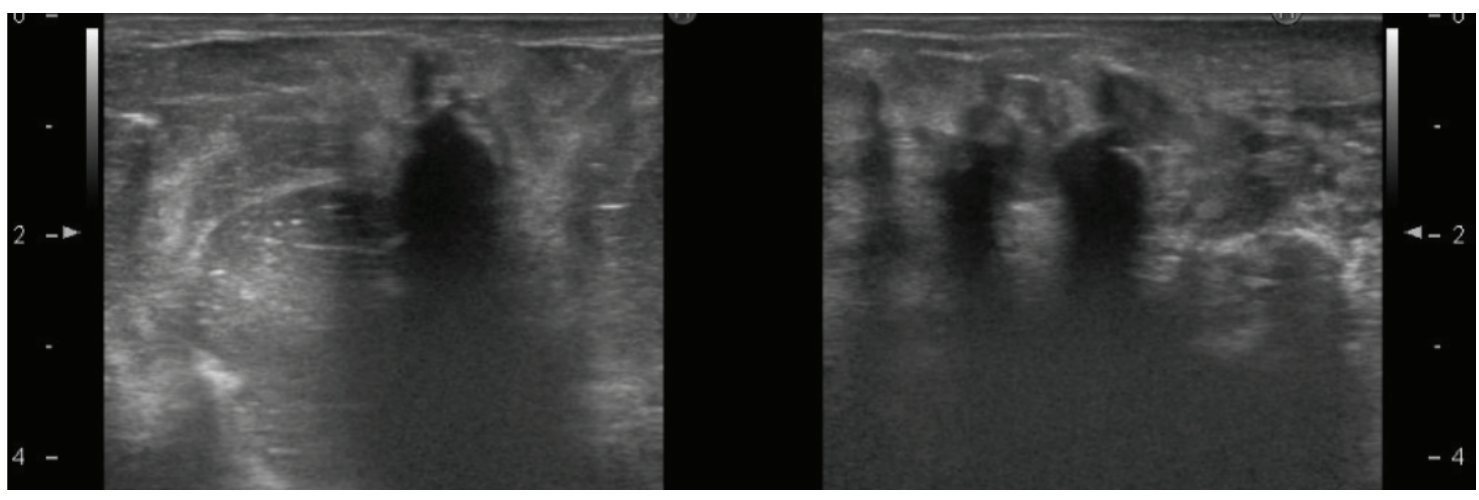

Figure 1. Ultrasound images of both breasts show ill-defined isoechoic masses with posterior acoustic shadow

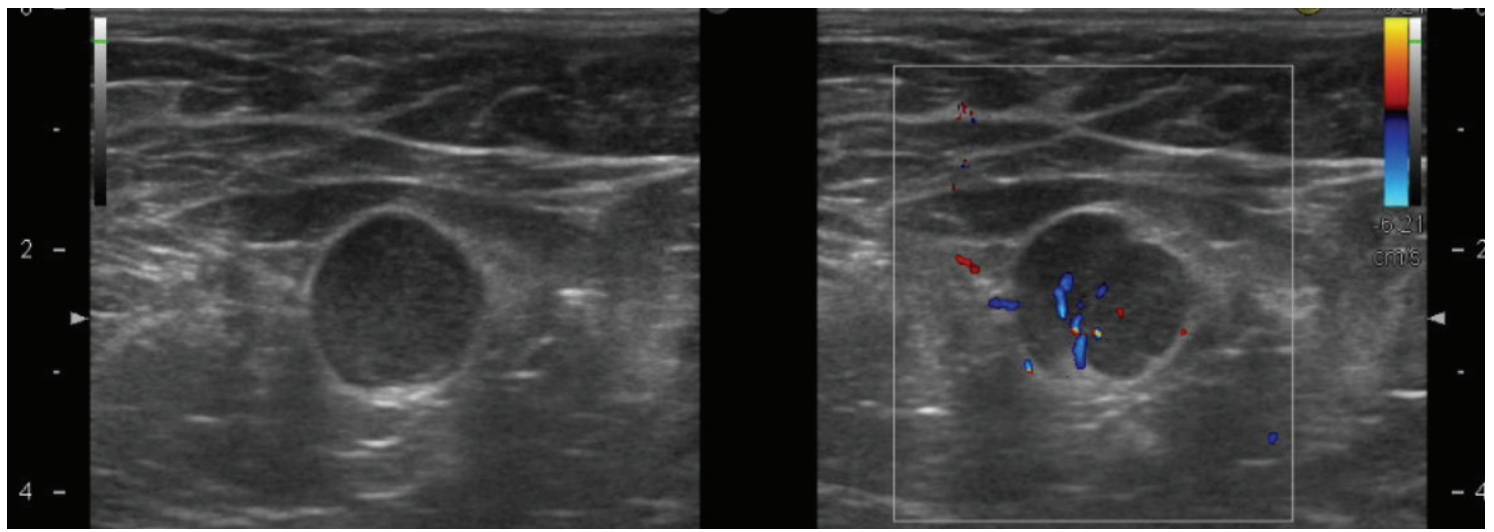

Figure 2. Ultrasound images show axillary lymph nodes enlargement, with a thick cortex 


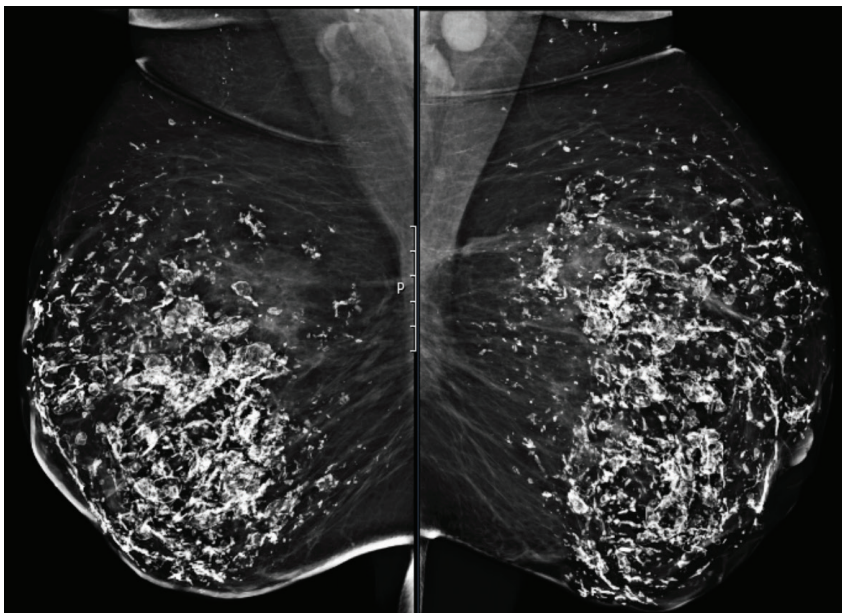

Figure 3. An MLO view of both breasts shows bilateral diffuse, coarse, and curvilinear calcifications, starting under the skin and scattered across the whole parenchyma of the breasts bilaterally

MLO: Mediolateral oblique

It is advisable to avoid biopsy because it may worsen the condition. This is possible if the diagnosis can be established with clinical and radiological features; the unusual mammographic calcification can be particularly helpful for this. However, fine needle aspiration (FNA) biopsy will be helpful if there is a doubt about the diagnosis or when the swelling is localized $(1,5,8)$. Accurate patient history and knowledge of the typical imaging appearance of LM may help prevent or minimize biopsy and surgical intervention $(8,9)$.

Histologically, most LM cases show lymphocyte-predominant inflammation, involving breast ducts, lobules, vessels, and adipose tissue, with hyaline fat necrosis being the most characteristic finding (10).

In our case, there were coarse and curvilinear calcifications on the mammogram and isoechoic masses with axillary lymphadenopathy on ultrasound.

LM should not be confused with breast carcinoma, idiopathic granulomatous mastitis, lymphoma, or other connective tissue diseases. The clinical features and histology help differentiate between these conditions $(2,11)$. However, LM may be exacerbated by surgical trauma so that needle core biopsy is preferred to open excisional biopsy. Indeed, the latter procedure has been reported to trigger a very painful progression of LM, eventually resulting in obligate mastectomy (4).

Antimalarial drugs are the primary medical treatment option for LM, while corticosteroids may also be used in combination or alone. Surgery should only be considered in patients with ongoing complaints despite appropriate medical treatment, because of the risk of additional exacerbations $(3,12)$.

In summary, we have reported an unusual case of LM in a female with known SLE. The patient presented with palpable breast masses. The bilateral breast calcification on mammography and isoechoic masses with posterior shadows on sonogram was mimicking a breast carcinoma. However, accurate patient history and knowledge of the typical imaging appearance on ultrasound and mammogram helped obtain a definitive diagnosis. Thus, awareness of the radiologic and clinical features of LM is essential to avoid unnecessary interventions such as biopsy and surgery that carries the potential for disease exacerbation.

Informed Consent: Written informed consent was obtained from the patient for publication of this case report.

Peer-review: Externally peer-reviewed.

\section{Authorship Contributions}

Surgical and Medical Practices: A.O., Ö.A.; Concept: A.O.; Design: A.O., H.A.E.; Data Collection and/or Processing: H.A.E., I.M.; Analysis and/or Interpretation: H.A.E, Ö.A.; Literature Searching: I.M.; Writing: H.A.E.

Conflict of Interest: The authors have no conflicts of interest to declare.

Financial Disclosure: The authors declared that this study has received no financial support.

\section{References}

1. Kinonen C, Gattuso P, Reddy VB. Lupus mastitis: an uncommon complication of systemic or discoid lupus. Am J Surg Pathol 2010; 34: 901-906. (PMID: 20410809) [Crossref]

2. Wani AM, Mohd Hussain W, Fatani MI, Shakour BA. Lupus mastitis peculiar radiological and pathological features. Indian J Radiol Imaging 2009; 19: 170-172. (PMID: 19881078) [Crossref]

3. Warne RR, Taylor D, Segal A, Irish A. Lupus mastitis: a mimicker of breast carcinoma. BMJ Case Rep 2011; 2011: bcr1120115066. doi:10.1136/ bcr.11.2011.5066 (PMID: 22669997) [Crossref]

4. Lucivero G, Romano C, Ferraraccio F, Sellitto A, De Fanis U, Giunta R, et al. Lupus mastitis in systemic lupus erythematosus: a rare condition requiring a minimally invasive diagnostic approach. Int J Immunopathol Pharmacol 2011; 24: 1125-1129. (PMID: 22230423) [Crossref]

5. Corrêa JAP, Djahjah MCR. Lupus mastitis as differential diagnosis of breast Mass. J Case Rep 2020; 10: 162-165. [Crossref]

6. Goulabchand R, Hafidi A, Van de Perre P, Millet I, Maria ATJ, Morel J, et al. Mastitis in autoimmune diseases: review of the literature, diagnostic pathway, and pathophysiological key players. J Clin Med 2020; 9: 958. (PMID: 32235676) [Crossref]

7. Varma R, Szilagyi S, Harshan M. Breast involvement in mixed connective tissue disease. Radiol Case Rep 2019; 14: 430-435. (PMID: 30701011) [Crossref]

8. Vineetha M, Palakkal S, Sobhanakumari K, Celine MI. Interchanging autoimmunity - lupus mastitis coexisting with systemic polyarteritis nodosa. Indian J Dermatol 2016; 61: 200-202. (PMID: 27057023) [Crossref]

9. Mosier AD, Boldt B, Keylock J, Smith DV, Graham J. Serial MR findings and comprehensive review of bilateral lupus mastitis with an additional case report. J Radiol Case Rep 2013; 7: 48-58. (PMID: 23372875) [Crossref]

10. Yan M, Bomeisl P, Gilmore H, Oduro K, Harbhajanka A. Lupus mastitis with predominant kappa-restricted plasma cell infiltration: report of a rare case. Surg Exp Pathol 2020; 3: 1-5. [Crossref]

11. Georgian-Smith D, Lawton TJ, Moe RE, Couser WG. Lupus mastitis: radiologic and pathologic features. AJR Am J Roentgenol 2002; 178 : 1233-1235. (PMID: 11959738)

12. Dandinoglu T, Dandin O, Akpak YK, Ergin T, Karadeniz M. Can lupus mastitis be treated surgically. Orthop Muscul Syst 2014; 3: 1000151. [Crossref] 